\title{
The influence of gender and cultural values on savoring in Korean undergraduates
}

\author{
Soyeon Kim · Fred B. Bryant
}

\begin{abstract}
The present study investigated antecedents of savoring beliefs and responses in a sample of South Korean college students. Historically, Korea has been strongly influenced by Chinese Confucianism, which emphasizes not only gender-role differentiation and patriarchal norms, but also the dampening of emotions as a culturally appropriate style of positive emotional regulation. We hypothesized that Korean females, relative to males, would reject traditional Asian cultural values in order to gain more empowerment, and would, as a result, report a greater capacity to savor positive experience. Confirming the hypotheses, Korean women, compared to men, reported stronger disagreement with traditional Asian values, greater overall savoring ability, greater capacity for cognitive elaboration, and less use of dampening and greater use of amplifying as savoring responses to positive events. Path analyses supported our hypothesized mediational model in which Korean women, relative to men, more strongly rejected traditional Asian values, which predicted less dampening (but only marginally greater amplifying). We conclude that among young Korean adults: (a) savoring is a relevant concept; (b) traditional Asian values tend to promote dampening of positive emotions; and (c) women more strongly reject traditional cultural values, tend to engage in less dampening and greater amplifying, and perceive greater savoring capacity, relative to men.
\end{abstract}

Keywords: savoring, gender, Confucianism, cross-cultural

\section{Introduction}

Although the field of psychology has traditionally focused on psychological distress and psychopathology (Bryant, 2003), the fast-growing field of positive psychology (Seligman \& Csikszentmihalyi, 2000) has integrated positive mental health and wellbeing to gain a more comprehensive understanding of adaptive human functioning. Paralleling the process of coping, through which people regulate the adverse consequences of distress, savoring has been recognized as a process through which people attend to positive experiences and engage in thoughts and behaviors that regulate positive feelings that arise from these experiences (Bryant, 1989; Bryant, 2003; Bryant, Chadwick, \& Kluwe, 2011; Bryant \& Veroff, 2007). In the present study, we investigate the influence of gender and traditional Asian values on savoring among young Korean adults.

\subsection{Savoring}

Bryant and Veroff (2007) described savoring as reflecting people's capacities to attend to and appreciate positive life experiences, and to regulate positive feelings in response to positive events. In other words, savoring processes can amplify or dampen the intensity of positive 
emotions, and can extend or shorten the duration of enjoyment derived from positive events. In explicating the concept of savoring, Bryant and Veroff (2007) distinguished savoring beliefs (i.e., self-evaluations of one's ability to regulate positive experience) from savoring responses (i.e., thoughts and behaviors in which people engage in reaction to positive experiences, and which regulate levels of consequent positive emotion, including happiness). Prospective longitudinal research indicates that savoring responses predict one's level of happiness in daily life (Jose, Lim, \& Bryant, 2012).

Developing and validating the Savoring Beliefs Inventory (SBI) as a measure of perceived ability to savor positive experiences, Bryant (2003) demonstrated that people hold savoring beliefs with respect to three temporal orientations reflecting the ability to derive enjoyment in the present by (a) anticipating future positive events before these events happen, (b) savoring the moment using specific cognitive and behavioral savoring strategies while positive events are unfolding, and (c) reminiscing about past positive events after these events have occurred. Using the SBI in American samples, Bryant (2003) found that, compared to men, women feel more capable of enjoying positive outcomes in all three temporal domains - that is, by anticipating upcoming positive events, savoring the moment, and reminiscing about positive memories. These gender differences in savoring beliefs have been found in a variety of Western countries, including the United States, Canada, and Australia (Bryant \& Veroff, 2007).

Developing and validating the Ways of Savoring Checklist (WOSC), which measures thoughts and behaviors in response to positive events, Bryant and Veroff (2007) identified ten different savoring subscales (i.e., Sharing with Others, Memory Building, Self-Congratulation, Comparing, Sensory-Perceptual Sharpening, Absorption, Behavioral Expression, Temporal Awareness, Counting Blessings, and Kill-Joy Thinking). Yet, the positive psychology literature currently lacks systematic research on savoring beliefs and savoring responses in Asian cultures in general and in Korean samples in particular.

\subsection{Savoring in Asian cultures}

Traditionally, collectivist Asian cultures have emphasized dampening of positive emotions, in contrast to individualistic Western cultures that have emphasized amplifying of positive emotions (Miyamoto \& Ma, 2011). East Asian individuals may seek to diminish the inner experience and outward display of positive and negative emotion due to cultural scripts involving dialectical beliefs, i.e., that reality is constantly changing - misery becomes happiness, and happiness becomes misery - and the balanced "middle way" is best (Miyamoto \& Ma, 2011). Within this cultural context, Korean individuals may tend to respond to positive events with what have been termed "kill-joy" thoughts (Beck, 1975; Bryant \& Veroff, 2007) that dampen positive emotions in Western samples.

Along these lines, cross-cultural research on the relationship between savoring and fear of happiness (Joshanloo, Lepshokova, Panyusheva, Natalia, Poon, Yeung et al., 2014) also indicates that people who believe happiness leads to negative consequences tend to engage in higher levels of dampening to regulate positive emotions. In a related vein, studying the relationship between emotional display rules and individualism versus collectivism among more than 5,000 respondents in 32 countries, Matsumoto, Yoo, Fontaine, Anguas-Wong, Ariola, Ataca et al. (2008) found that collectivist countries had lower expressivity norms for positive emotions, compared to individualistic countries. Likewise, Miyamoto and Ma (2011) concluded that cultural scripts shape emotion regulation in Japan, such that Japanese are less inclined than Americans to amplify positive emotions. Considered together, this relevant research suggests that dampening responses may be more prevalent than amplifying responses in Korean samples. 


\subsection{Gender differences in emotional experiences and savoring}

In comparing the emotions of men and women, Grossman and Wood (1993) have used social role theory to explain observed gender differences in emotional experience. In general, research has demonstrated that women report more intense emotions than men and are more likely to seek out and express emotional experiences (Eagly \& Wood, 1982). These gender differences in emotion may be a consequence of traditional sex roles, such as the caretaker role of women. For example, relative to men, women may show greater emotional sensitivity and expressiveness because women's traditional caretaker role required emotion-related skills, which are associated with beliefs and expectations that women should be more responsive to their own and others' emotions (Eagly \& Wood, 1982). Thus, gender differences in emotional experiences may have arisen from differential social beliefs and expectations regarding male and female roles (Grossman \& Wood, 1993).

\subsection{Confucianism and savoring in Korean culture}

Traditional Asian values may have a particularly strong influence in Korean culture. According to Kalton (1994), "It is almost a commonplace in East Asian studies" that Korea is and was "the 'most Confucian' of East Asian societies" (p. 239). The last and longest-lived Chosŏn Dynasty (1392-1910) prided itself on adherence to Confucian norms, which dictated propriety and harmonious attachment to self and others (Joshanloo, 2014) and allowed Korean women to have status and authority only within the domestic arena (Deuchler, 1993). Although South Korea has over the past few decades experienced "conflict between traditional values and the Confucian heritage on the one hand and Western influence through economic and social changes on the other" (Park \& Cho, 1995, p. 132), sociologists have argued that Confucian values continue to exert a stronger influence on South Korean culture than on other East Asian cultures.

What implications does the particularly strong influence of Confucian values on South Korean culture have for the ways of savoring among South Koreans as opposed to other East Asians? We might expect South Koreans to be especially predisposed to conceive of savoring in ways that are consistent with the Confucian notion of propriety and the dampening of emotions. Thus, we hypothesized that in a Korean sample, traditional Asian values would predict a savoring style of greater dampening. Because rejecting traditional Asian values does not necessarily imply greater endorsing of Western values, we also expected that rejecting traditional Asian values would predict lower levels of dampening but not necessarily higher levels of Western-style amplifying of positive emotions.

\subsection{Gender and cultural values in Korea}

Although social psychologists have argued that patriarchal social structure is not a universal tendency (Wood \& Eagly, 2002), research has suggested that men generally have more social power than women across many cultures (Rosaldo \& Lamphere, 1974). Throughout its long history, Chinese Confucianism, which emphasizes strict gender-role differentiation and patriarchal social norms, has influenced many Asian countries (Kim, 1997). As a result, traditional Korean cultural values have also been embedded in these gender-differentiated Confucian norms, and historically, it was typical for Korean families to prefer sons over daughters (Deuchler, 1993).

Over time, however, education and globalization may alter such historical customs. According to Stacki and Monkman's (2003) process model of empowerment and change, education generally facilitates changes in the status quo and promotes gender equity. Women 
have traditionally been denied access to higher education in traditional Korean society, as was historically the case in Western societies. As a result of the pervasive influence of globalization, Western values have increasingly challenged traditional Korean values, especially among younger generations. Contrary to traditional Korean values, Western values accentuate individual achievement and independence and emphasize gender equity. Living in an era of conflict between traditional and new Westernized values, Korean males and females may differentially react by either accepting or rejecting traditional values. For example, Korean females relative to Korean males may be more likely to reject traditional Asian values because Korean females might gain more from the empowerment that rejecting these values provides, whereas Korean males derive greater power from the status quo within the context of Korean traditional values. Based on this reasoning, we hypothesized an integrative path model in which Korean females (relative to males) would more strongly reject traditional Asian values, which would predict lower levels of dampening for females.

\subsection{The purpose of the current study}

This study aims to compare males' and females' savoring beliefs and savoring responses in Korean culture. Although Bryant and Veroff's (2007) book on savoring was translated into Korean in 2010 (Kwon, Lim, \& Ha, 2010), the concept of savoring is new to Korean culture and has not been studied among Korean samples. Because the notion of savoring has been conceptualized and investigated largely within Western culture, it is important to understand Koreans' savoring beliefs and savoring responses from a Korean cultural perspective, framed in terms of traditional Asian cultural values.

Hypothesis 1. We hypothesized that Korean women, compared to Korean men, would more strongly reject traditional Asian values, as assessed by a Korean version of the Asian Values Scale (Kim, Atkinson, \& Yang, 1999).

Hypothesis 2. We hypothesized that Korean women would endorse stronger savoring beliefs (i.e., a greater savoring capacity) than would Korean men.

Hypothesis 3. We hypothesized that Korean women, compared to Korean men, would report less dampening and greater amplifying in response to positive events.

Hypothesis 4. We hypothesized an indirect effect linking gender to Asian values, and Asian values to savoring responses. In particular, we predicted a two-path mediational effect in which Korean females (relative to males) would more strongly reject traditional Asian values, which in turn would predict lower levels of dampening in response to positive events.

\section{Method}

\subsection{Participants}

Survey data were collected from 296 Korean participants in summer 2012 in Seoul, South Korea. However, survey data for only 288 respondents were analyzed, due to missing data for $2.7 \%$ of the sample. Participants were undergraduate students of four different Korean universities, and data collection at these various universities was approved by the IRB at Loyola University Chicago. Participants were recruited from two large public and two large private universities in the metropolitan area of Seoul, South Korea. Participants provided informed consent and had to be at least 18 years of age to take part in the research. 


\subsection{Procedures}

To check the equivalence of meaning in cross-cultural translation, we adopted a back-translation method (Brislin, 1970), through which all of the original English measures were translated into Korean by the bilingual researcher (SK) and then back-translated to English afterward by a second bilingual Korean person. During data collection, the researcher obtained completed informed consent forms at the beginning of the study, and asked participants to fill out the survey questionnaire either in class or later at the library. Participants were informed that the survey was anonymous and their survey responses would remain confidential. In exchange for voluntary participation in the study, a free ballpoint pen was offered to each participant. At the end of the study, the researcher debriefed participants and described the research hypotheses.

\subsection{Measures}

Cultural values. The Asian Values Scale (AVS; Kim, Atkinson, \& Yang, 1999) was used to assess cultural values associated with Korean respondents' ethnic and cultural beliefs, including conformity to norms, family recognition through achievement, emotional self-control, collectivism, humility, and filial piety. The AVS consists of 36 statements to which respondents indicate their level of agreement, using a seven-point scale ranging from (1) strongly disagree to (7) strongly agree. Research supports the internal consistency and test-retest reliability of the AVS, as well as its structural and construct validity as a measure of traditional Asian cultural values (Kim et al., 1999; Kim, Yang, Atkinson, Wolfe, \& Hong, 2001).

Savoring beliefs. The Savoring Beliefs Inventory (SBI; Bryant, 2003) was used to measure individuals' perceived capacity to savor positive experience in terms of their ability to derive enjoyment through anticipating future positive events, appreciating positive events in the moment, and reminiscing about past positive events. The SBI consists of 24 statements that respondents rate in terms of how true these statements are for them personally, using a sevenpoint scale ranging from (1) strongly disagree to (7) strongly agree. Eight SBI items assess futurefocused savoring through anticipation, eight items assess present-focused savoring of the moment, and eight items assess past-focused savoring through reminiscence, and within each temporal domain, half of the items reflect the endorsement of savoring ability, and half reflect the rejection of savoring ability (Bryant, 2003). Strong evidence supports the SBI's internal consistency and test-retest reliability, as well as its structural, construct, and criterion validity as a measure of savoring capacity across a wide age range (Bryant, 2003; Bryant \& Veroff, 2007).

Savoring responses. The Ways of Savoring Checklist (WOSC; Bryant \& Veroff, 2007) was used to measure participants' savoring responses. The 56 closed-ended WOSC items assess cognitive and behavioral responses to a recent positive event of the respondent's choice. Respondents rate each savoring response in terms of the degree to which it applies to what they thought and did during the positive event, using a seven-point scale ranging from (1) definitely doesn't apply, to (4) applies somewhat, to (7) definitely applies. The WOSC provides 10 subscale scores reflecting different types of cognitive and behavioral savoring strategies people use to regulate good feelings during positive experiences: Sharing with Others, Memory Building, SelfCongratulation, Sensory-Perceptual Sharpening, Comparing, Absorption, Behavioral Expression, Temporal Awareness, Counting Blessings, and Kill-Joy Thinking. There is evidence to support the internal consistency reliability of the WOSC, as well as its structural, construct, and prospective criterion validity as a measure of savoring responses to positive events (Bryant \& Veroff, 2007; Jose et al., 2012; Smith \& Bryant, 2012, 2017). 


\subsection{Establishing item sets}

Although the field of psychology is largely based on Western traditions that emphasize liberal individualism (Joshanloo, 2014), theories grounded in Western perspectives are not necessarily generalizable to Eastern cultures (Uchida \& Kitayama, 2009). Whereas Easterners' emotional experiences tend to emphasize interdependence, collectivism, and harmonious relationships with others, Westerners' emotional experiences typically reflect a greater emphasis on independence and individualism (Uchida, Norasakkunkit, \& Kitayama, 2004). Because Korea is an East Asian culture, Koreans may report savoring beliefs and may savor positive experiences in ways that differ from those of Westerners. For this reason, we chose to construct measurement models of savoring beliefs and savoring responses based on exploratory factor analyses of our Korean data, rather than simply adopting measurement structures developed using Western samples.

\section{Results}

\subsection{Constructing composite subscales}

To develop composite measures of factors underlying responses to each self-report instrument that were appropriate for the Korean sample, we used principal axis factoring (PAF) with promax rotation to allow latent factors to be correlated and maximize the common variance that the factors explained (Thompson, 2004). Horn's (1965) parallel analysis, which is considered the optimal method for determining the most appropriate number of underlying factors (Dinno, 2009), was used to identify the underlying structures and subscales for each self-report instrument. In particular, we used PARAN, a plug-in of the Visual Statistics System (Young, Valero-Mora, \& Friendly, 2006), to conduct nonparametric Monte Carlo parallel analysis of responses to each instrument by generating 1,000 random data sets each with $N=288$, based on multivariate permutations of the sample data. In interpreting factors and constructing composite subscales, we retained items with loadings $\geq .40$ (Matsunaga, 2010).

Asian Values Scale. From PAF with promax rotation and the parallel analysis, four factors were extracted for the set of 36 items constituting the Asian Values Scale. However, based on recommendations in the factor analysis literature (Velicer \& Fava, 1998), we decided to drop the third and fourth factors on which only two items loaded $\geq .40$, and we thus analyzed only the first two PAF factors (see Table 1 below).

The first factor, labeled "Conformity to Collectivist Social Norms," consisted of eight items (Cronbach's $\alpha=.76)$, e.g., "One should think about one's group before oneself." The second factor, labeled "Rejection of Traditional Asian Values," was composed of seven items $(\alpha=.75)$, e.g., "One should be able to question a person in an authority position."

Savoring Beliefs Inventory. Rather than adopting the SBI subscales developed using Western samples, we sought to identify SBI dimensions that were relevant for Korean respondents. The results of PAF with promax rotation suggested that three reliable factors underlie responses to the 24 items constituting the Korean version of the Savoring Beliefs Inventory (SBI; Bryant, 2003), as also reported in Table 1, and the parallel analysis confirmed the appropriateness of retaining three factors. The first factor, labeled "Endorsement of Savoring," consisted of 12 items - e.g., "I enjoy looking back on happy times from my past," "I know how to make the most of a good time," "Before a good thing happens, I look forward to it in ways that give me pleasure in the present" - reflecting the belief that one can amplify positive emotional experiences through retrospective, concurrent, or prospective savoring $(\alpha=.87)$. 
Table 1a. Results of separate, exploratory principal axis factor analyses with promax rotation for the Asian Value Scale, Savoring Beliefs Inventory, and Ways of Savoring Checklist $(N=288)$

\begin{tabular}{|c|c|c|}
\hline \multirow{2}{*}{ Asian Values Scale (AVS) items } & \multicolumn{2}{|c|}{ Item Factor Loadings } \\
\hline & Factor 1 & Factor 2 \\
\hline \multicolumn{3}{|l|}{ 1. Conformity to Collectivist Social Norms: } \\
\hline Avoid bringing displeasure to ancestors & .75 & -.15 \\
\hline Follow familial and social expectations & .67 & -.19 \\
\hline Should not make waves & .54 & -.00 \\
\hline Do not disgrace family reputation & .53 & .40 \\
\hline Should think about group before oneself & .52 & .26 \\
\hline Regard individual achievements as family's achievements & .44 & .14 \\
\hline Do not leave parents in retirement homes & .43 & -.07 \\
\hline Do not deviate from familial and social norms & .42 & .23 \\
\hline \multicolumn{3}{|l|}{ 2. Rejection of Traditional Asian Values: } \\
\hline One doesn't have to focus on studies & .01 & .60 \\
\hline Young people can confront their elders & -.09 & .58 \\
\hline Elders may not have more wisdom than young people & .02 & .58 \\
\hline Educational and career achievements are not the top priority & -.08 & .57 \\
\hline Parents' love should be openly expressed & .23 & .53 \\
\hline Family's reputation is not the primary concern & .17 & .44 \\
\hline One should be able to question an authority figure & -.07 & .42 \\
\hline Percentage of variance & 14.43 & 11.55 \\
\hline Eigenvalue & 5.20 & 4.16 \\
\hline
\end{tabular}


Table 1b. Results of separate, exploratory principal axis factor analyses with promax rotation for the Asian Value Scale, Savoring Beliefs Inventory, and Ways of Savoring Checklist $(N=288)$

\begin{tabular}{|c|c|c|c|}
\hline \multirow{2}{*}{ Savoring Beliefs Inventory (SBI) items } & \multicolumn{3}{|c|}{ Item Factor Loadings } \\
\hline & Factor 1 & Factor 2 & Factor 3 \\
\hline \multicolumn{4}{|l|}{ 1. Endorsement of Savoring: } \\
\hline Make myself feel good by remembering pleasant events & .74 & -.11 & .08 \\
\hline Like to store memories of fun times & .71 & -.33 & .21 \\
\hline Enjoy looking back on happy times & .64 & -.18 & .31 \\
\hline Know how to make the most of a good time & .62 & -.05 & -.21 \\
\hline Easy to rekindle the joy from pleasant memories & .60 & -.10 & -.11 \\
\hline Feel good by imagining happy moment before it happens & .56 & .14 & -.07 \\
\hline Make my enjoyment of a happy time last longer & .56 & -.04 & .11 \\
\hline Enjoy pleasant events in my mind before they occur & .55 & .25 & .02 \\
\hline Feel fully able to appreciate good things & .55 & -.21 & -.05 \\
\hline Easy for me to enjoy myself & .51 & .21 & -.27 \\
\hline Get pleasure by looking forward to good things & .44 & .22 & -.28 \\
\hline Feel the joy of anticipation & .44 & .24 & -.04 \\
\hline \multicolumn{4}{|l|}{ 2. Rejection of Cognitive Elaboration: } \\
\hline Thinking about past good times is a waste of time & .00 & .73 & .18 \\
\hline Once a fun time is over, do not think about it & .04 & .72 & -.03 \\
\hline Don't like to look back at good times & -.25 & .67 & -.12 \\
\hline Can't capture the joy of happy moments & .02 & .50 & .25 \\
\hline Hard to enjoy myself & -.04 & .48 & .36 \\
\hline Anticipating upcoming good events is a waste of time & .00 & .40 & .19 \\
\hline \multicolumn{4}{|l|}{ 3. Rejection of Positive Affective Consequences: } \\
\hline Reminiscing about pleasant memories makes me sad & .09 & -.05 & .67 \\
\hline Don't enjoy things as much as I should & -.05 & .18 & .64 \\
\hline Hard for me to hang onto a good feeling & -.07 & .16 & .61 \\
\hline Anticipating a pleasant event makes me uncomfortable & .05 & .21 & .47 \\
\hline Percentage of variance & 28.29 & 13.32 & 8.11 \\
\hline Eigenvalue & 6.79 & 3.20 & 1.95 \\
\hline
\end{tabular}


Table 1c. Results of separate, exploratory principal axis factor analyses with promax rotation for the Asian Value Scale, Savoring Beliefs Inventory, and Ways of Savoring Checklist $(N=288)$

\begin{tabular}{|c|c|c|c|c|c|c|}
\hline \multirow{2}{*}{ The Ways of Savoring Checklist (WOSC) items } & \multicolumn{6}{|c|}{ Item Factor Loadings } \\
\hline & Factor 1 & Factor 2 & Factor 3 & Factor 4 & Factor 5 & Factor 6 \\
\hline \multicolumn{7}{|l|}{ 1. Broadband Dampening: } \\
\hline Withdrew my feelings & .72 & -.06 & -.10 & .09 & .01 & -.03 \\
\hline Thought the positive event wasn't as good as I'd hoped for & .70 & -.34 & .03 & .19 & -.03 & .11 \\
\hline Thought something that made me feel guilty & .67 & .05 & .03 & -.18 & .03 & -.06 \\
\hline Reminded myself of other places I should be & .66 & -.13 & .02 & -.05 & .11 & .06 \\
\hline Focused on the future & .56 & .17 & -.02 & .00 & .02 & -.07 \\
\hline Thought about other problems I still had to face & .54 & -.06 & .19 & -.21 & .02 & .09 \\
\hline Reminded myself the positive event would be over soon & .51 & -.09 & .40 & -.03 & -.11 & -.06 \\
\hline Told myself why I didn't deserve this positive event & .49 & .13 & -.04 & .09 & -.03 & -.01 \\
\hline Compared myself to others & .46 & .20 & .18 & -.19 & .01 & .15 \\
\hline Tried to speed up and move more quickly & .45 & -.16 & .20 & .13 & .02 & .05 \\
\hline \multicolumn{7}{|l|}{ 2. Broadband Amplifying: } \\
\hline Thought about what a triumph the positive event was & -.10 & .74 & .05 & -.08 & .08 & -.12 \\
\hline Gave thanks for my good fortune & .07 & .68 & -.09 & .15 & -.03 & -.17 \\
\hline Reminded myself how lucky I was & -.13 & .64 & -.06 & .10 & -.12 & .11 \\
\hline Reminded myself what a relief it was & -.19 & .55 & .08 & .03 & -.07 & -.06 \\
\hline Told myself how proud I was & .17 & .54 & -.16 & .03 & .02 & .16 \\
\hline Thought about what a lucky person I am to have good things & .09 & .49 & -.05 & .15 & -.10 & .01 \\
\hline Told myself how impressed others must be & .29 & .48 & -.09 & .05 & .18 & -.03 \\
\hline \multicolumn{7}{|l|}{ 3. Awareness of Temporal Fleetingness \& Memory Building: } \\
\hline Reminded myself that I must enjoy it now & .09 & -.18 & .71 & -.05 & -.03 & .00 \\
\hline Thought about how fast the time was passing & .22 & .07 & .66 & -.01 & -.12 & -.17 \\
\hline Reminded myself that nothing lasts forever & .08 & -.03 & .65 & .07 & .06 & -.08 \\
\hline Tried to memorize my surroundings & -.03 & .23 & .49 & .03 & -.04 & .10 \\
\hline Thought about how things might never be this good again & .14 & .27 & .48 & -.04 & -.01 & -.09 \\
\hline Reminded myself how transient this moment was & .07 & .01 & .44 & .20 & .01 & -.07 \\
\hline Thought about what a good time I was having & -.16 & .27 & .43 & .11 & .01 & .05 \\
\hline Took photographs with a camera & .13 & -.22 & .43 & .12 & .16 & .12 \\
\hline Took mental photographs & -.23 & .32 & .41 & .00 & .20 & -.02 \\
\hline
\end{tabular}


Table 1d. Results of separate, exploratory principal axis factor analyses with promax rotation for the Asian Value Scale, Savoring Beliefs Inventory, and Ways of Savoring Checklist $(N=288)$

\begin{tabular}{|c|c|c|c|c|c|c|}
\hline \multirow{2}{*}{ The Ways of Savoring Checklist (WOSC) items } & \multicolumn{6}{|c|}{ Item Factor Loadings } \\
\hline & Factor 1 & Factor 2 & Factor 3 & Factor 4 & Factor 5 & Factor 6 \\
\hline \multicolumn{7}{|l|}{ 4. Sensory-Perceptual Sharpening \& Absorption: } \\
\hline Tried to focus on certain sensory properties & .19 & -.22 & -.07 & .62 & .21 & -.01 \\
\hline Thought only about the present & -.19 & .03 & .01 & .62 & .10 & -.01 \\
\hline Relaxed to try to become more absorbed in the event & -.29 & .09 & .18 & .56 & -.04 & -.06 \\
\hline Blocked out distractions & .18 & .05 & .09 & .56 & .05 & -.23 \\
\hline Tried to take in every sensory property of the event & -.15 & .04 & .03 & .51 & -.05 & .37 \\
\hline Took in the moment & .06 & .25 & .05 & .48 & -.03 & -.11 \\
\hline Tried to become more alert & .31 & .23 & -.19 & .43 & .04 & -.02 \\
\hline Tried not to look too far ahead & .20 & .25 & .01 & .41 & -.09 & -.03 \\
\hline Tried to slow down & .29 & .07 & .10 & .41 & -.13 & -.02 \\
\hline \multicolumn{7}{|l|}{ 5. Behavioral Expression: } \\
\hline Made verbal expressions of excitement & -.04 & -.03 & .04 & -.02 & .87 & -.08 \\
\hline Touched myself & .07 & .01 & -.09 & -.08 & .85 & -.04 \\
\hline Made other verbal sounds of appreciation & .00 & -.04 & .12 & .11 & .68 & -.11 \\
\hline Showed physical expressions of energy & .11 & -.04 & -.18 & .20 & .63 & .06 \\
\hline \multicolumn{7}{|l|}{ 6. Sharing with Others: } \\
\hline Thought about sharing this later with others & .11 & -.04 & -.08 & -.06 & -.14 & .94 \\
\hline Looked for others to share this with & .02 & .01 & -.03 & -.09 & .00 & .77 \\
\hline Talked to others about this good feeling & -.06 & .23 & -.01 & -.13 & .20 & .50 \\
\hline Percentage of variance & 23.27 & 8.52 & 4.91 & 4.37 & 3.73 & 3.05 \\
\hline Eigenvalue & 13.03 & 4.77 & 2.75 & 2.45 & 2.09 & 1.71 \\
\hline
\end{tabular}

Note: Factor loadings $\geq .40$ (Matsunaga, 2010) are bolded. Because AVS Factors 3 and 4 and WOSC Factor 7 had only two items with loadings $\geq .40$, they were excluded from the analyses and omitted from this table (see Velicer \& Fava, 1998). Due to copyright restrictions, the items in this table have been paraphrased from the original wording so as to convey item content without reporting exact verbatim wording. 
The second factor, labeled "Rejection of Cognitive Elaboration," contained six items - e.g., "For me, once a fun time is over and gone, it's best not to think about it," "I can't seem to capture the joy of happy moments," "For me, anticipating what upcoming good events will be like is basically a waste of time" - reflecting the rejection of cognitive elaboration as a way to savor positive experiences in the past, present, or future $(\alpha=.83)$.

The third factor, labeled "Rejection of Positive Affective Consequences," included four items-e.g., "When I reminisce about pleasant memories, I often start to feel sad or disappointed," "It's hard for me to hang onto a good feeling for very long," "When I think about a pleasant event before it happens, I often start to feel uneasy or uncomfortable" - reflecting the belief that one can amplify or prolong positive feelings in relation to the past, present, or future $(\alpha=.73)$.

The Ways of Savoring Checklist. Rather than adopting the WOSC subscales developed using Western samples, we sought to identify ways of savoring that were applicable to Korean respondents. Both PAF with promax rotation and the parallel analysis revealed that seven dominant factors underlie responses to the 56 items constituting the Korean version of the WOSC. However, the seventh factor consisted of only two items with loadings $\geq .40$, and thus was excluded from our analyses (see Velicer \& Fava, 1998). As also seen in Table 1, the first factor, "Broadband Dampening," consisted of ten items - e.g., "I withdrew and inhibited my feelings" reflecting kill-joy thinking as a general dampening strategy in response to positive events $(\alpha=$ $.84)$.

The second factor, "Broadband Amplifying," included seven items - e.g., "I reminded myself how lucky I was to have this good thing happen to me" - reflecting cognitive savoring responses that serve to amplify positive emotions $(\alpha=.81)$.

The third factor, labeled "Awareness of Temporal Fleetingness and Memory Building," included nine items-e.g., "I thought how I wished this moment could last - reminded myself how I must enjoy it now because it would soon be over" - reflecting perceptions of the scarcity of time during positive events and the active encoding of positive situational details to preserve these experiences for later recall $(\alpha=.83)$.

The fourth factor, labeled "Sensory-Perceptual Sharpening and Absorption," consisted of nine items-e.g., "I tried to take in every sensory property of the event (sights, sounds, smells, etc.)" - reflecting active efforts to block out distracting stimuli in order to become more fully engrossed in the moment $(\alpha=.83)$.

The fifth factor, labeled "Behavioral Expression," included four items-e.g., "I jumped up and down, ran around or showed other physical expressions of energy" - reflecting the physical display of positive emotions $(\alpha=.83)$.

The sixth factor, labeled "Sharing with Others," consisted of three items-e.g., "I thought about sharing the memory of this later with other people"-reflecting spontaneous communication of positive emotions to other people $(\alpha=.79)$.

\subsection{Descriptive statistics}

Table 2 reports the results of descriptive analyses $(N=288)$, including variable means, standard deviations, and intercorrelations. Correlations between specific subscales provide evidence concerning the convergent validity of these particular measures. For instance, the first SBI subscale, "Endorsement of Savoring Beliefs," is significantly and positively correlated with the second WOSC subscale, "Amplification of Positive Emotional Experiences," $r=.44, p<.001$, as would be expected if both subscales in fact reflect tendencies to intensify and prolong positive emotional experiences. 
Table 2. Intercorrelations and descriptive statistics for SBI subscales, WOSC subscales, AVS subscales, and gender $(N=288)$

\begin{tabular}{|c|c|c|c|c|c|c|c|c|c|c|c|}
\hline & 1 & 2 & 3 & 4 & 5 & 6 & 7 & 8 & 9 & 10 & 11 \\
\hline 1. SBI1 Endorsement of Savoring & .87 & & & & & & & & & & \\
\hline 2. SBI2 Rejection of Cognitive Elaboration & -.31 & .83 & & & & & & & & & \\
\hline 3. SBI3 Rejection of Positive Affective Consequences & -.28 & .50 & .73 & & & & & & & & \\
\hline 4. WOSC1 Broadband Dampening & .02 & .59 & .47 & .84 & & & & & & & \\
\hline 5. WOSC2 Broadband Amplifying & .44 & .01 & .05 & .34 & .81 & & & & & & \\
\hline $\begin{array}{l}\text { 6. WOSC3 Awareness of Temporal Fleetingness \& } \\
\text { Memory Building }\end{array}$ & .33 & .01 & .25 & .37 & .51 & .83 & & & & & \\
\hline 7. WOSC4 Sensory-Perceptual Sharpening \& Absorption & .40 & .23 & .12 & .46 & .53 & .55 & .83 & & & & \\
\hline 8. WOSC5 Behavioral Expression & .16 & .01 & .08 & .28 & .35 & .35 & .36 & .83 & & & \\
\hline 9. WOSC6 Sharing with Others & .31 & -.14 & .01 & .05 & .35 & .31 & .16 & .37 & .79 & & \\
\hline 10. AVS1 Conformity to Collectivist Social Norms & .07 & .19 & -.02 & .18 & .07 & .05 & .08 & -.16 & -.10 & .76 & \\
\hline 11. AVS2 Rejection of Traditional Asian Values & .13 & -.45 & -.12 & -.32 & -.05 & -.02 & -.17 & .01 & .15 & -.27 & .75 \\
\hline 12. Gender & .15 & -.35 & -.02 & -.16 & .17 & .16 & -.03 & .10 & .20 & -.26 & -.25 \\
\hline Mean & 5.05 & 2.58 & 3.45 & 3.04 & 4.51 & 4.48 & 3.98 & 4.13 & 5.12 & 3.85 & 5.24 \\
\hline$S D$ & .91 & 1.15 & 1.25 & 1.14 & 1.21 & 1.25 & 1.17 & 1.62 & 1.39 & .96 & .87 \\
\hline
\end{tabular}

Note: SBI = Savoring Beliefs Inventory. WOSC = Ways of Savoring Checklist. AVS = Asian Values Scale. SD = standard deviation. All subscale scores were constructed by averaging scores for constituent items. $|r s|=.116$ are significant at two-tailed $p=.05$. $|r s|=.152$ are significant at two-tailed $p=.01$. $|r s|=.193$ are significant at two-tailed $p=.001$. Gender $(0=$ male, $1=$ female $)$. Cronbach's alpha for each subscale is tabled in the diagonal. 
In addition, there is a strong negative correlation between the first SBI subscale, "Endorsement of Savoring Beliefs," and the first WOSC subscale, "Dampening of Positive Emotional Experiences," $r=-.59, p<.001$, as would be expected if these subscales actually reflect what they are presumed to measure.

\subsection{Hypothesis Testing}

Hypothesis 1: Gender differences in Asian values. As hypothesized, Korean women scored higher on the Rejection of Traditional Asian Values subscale of the AVS than did Korean men, $t(286)=4.44, p<.001$, Cohen's $d=0.53, r=.25$. On the other hand, Korean men scored higher on the Conformity to Collectivist Social Norms subscale of the AVS than did Korean women, $t(286)=4.54, p<.001, d=0.54, r=.26$.

Hypothesis 2: Gender differences in savoring beliefs. Supporting the second hypothesis, Korean women scored higher on the Endorsement of Savoring Beliefs subscale of the SBI than did Korean men, $t(286)=2.54, p<.05, d=0.30, r=.15$, whereas Korean men scored higher on the Rejection of Cognitive Elaboration subscale of the SBI than did Korean women, $t(286)=6.28, p<.001, d=0.79, r=.37$. Contrary to Hypothesis 2 , however, there was no significant gender difference on the SBI subscale assessing Rejection of Positive Affective Consequences, $t(286)=0.28, p=.78, d=0.03, r=.02$.

Hypothesis 3: Gender differences in savoring responses. Consistent with Hypothesis 3, Korean men reported higher levels of WOSC Broadband Dampening than did Korean women, $t(286)=2.63, p<.01, d=0.33, r=.16$; and Korean women, in contrast, reported a greater use of WOSC Broadband Amplifying than did Korean men, $t(286)=2.99, p<.01, d=0.35$, $r=.17$. Along with the broadband amplifying strategies, two narrowband amplifying strategies revealed the same hypothesized pattern of gender differences. Specifically, Korean women, compared to Korean men, reported greater use of the WOSC savoring strategies of: (1) Awareness of Temporal Fleetingness and Memory-Building, $t(286)=2.65$, $p<.01, d=0.31, r=.15$; and (2) Sharing with Others, $t(286)=3.45, p<.01, d=0.41, r=.20$. Contrary to Hypothesis 3, no significant gender differences emerged for the other two narrowband WOSC amplifying strategies: (1) Sensory-Perceptual Sharpening and Absorption, $t(286)=0.46, p=.65, d=0.05, r=.03$; and (2) Behavioral Expression, $t(286)=$ $1.77, p<.08, d=0.21, r=.10$ (see Table 3).

Hypothesis 4: Indirect effects of Asian values on savoring responses. To estimate the hypothesized mediational effect, we employed the Preacher and Hayes (2008) SPSS bootstrap macro program using bias-corrected bootstrapping with 5,000 resamples, as well as a normal-theory Sobel test of the significance of the indirect effect (see Table 4). As hypothesized, Korean participants' cultural values mediated the influence of gender differences on savoring responses.

In particular, participants' rejection of traditional Asian values significantly mediated the effects of gender on dampening (see Figure 1 below), which represents the most dominant and culturally appropriate savoring response among Korean participants: estimated indirect effect $=-0.17, S E=0.05, Z=3.38, p<.001$, bias corrected $95 \%$ CI [-0.31, -0.08 ]. 
Table 3. Gender comparison of mean levels of Asian values, savoring beliefs, and savoring responses

\begin{tabular}{|c|c|c|c|c|c|c|c|c|c|}
\hline \multirow[b]{2}{*}{ Dependent Measures } & \multicolumn{2}{|c|}{$\begin{array}{c}\text { Males } \\
(N=134)\end{array}$} & \multicolumn{2}{|c|}{$\begin{array}{l}\text { Females } \\
(N=154)\end{array}$} & \multicolumn{5}{|c|}{ Inferential Statistical Tests } \\
\hline & $M$ & $S D$ & $M$ & $S D$ & $t$ & $d f$ & $p$ & $\begin{array}{c}\text { Cohen's } \\
d\end{array}$ & $\begin{array}{l}\text { Effect- } \\
\text { size } r\end{array}$ \\
\hline \multicolumn{10}{|l|}{ Asian Value Scale (AVS) Subscales: } \\
\hline Conformity to Collectivist Social Norms & 4.11 & 1.00 & 3.61 & 0.86 & 4.54 & 286 & $<.001$ & 0.54 & .26 \\
\hline Rejection of Traditional Asian Values & 5.00 & 0.90 & 5.45 & 0.79 & 4.44 & 286 & $<.001$ & 0.53 & .25 \\
\hline \multicolumn{10}{|l|}{ Savoring Beliefs Inventory (SBI) Subscales: } \\
\hline Endorsement of Savoring & 4.91 & 0.85 & 5.18 & 0.95 & 2.54 & 286 & .012 & 0.30 & .15 \\
\hline Rejection of Cognitive Elaboration & 3.01 & 1.19 & 2.20 & 0.96 & 6.28 & $286^{+}$ & $<.001$ & 0.79 & .37 \\
\hline Rejection of Positive Affective Consequences & 3.48 & 1.26 & 3.44 & 1.24 & 0.28 & 286 & .783 & 0.03 & .02 \\
\hline \multicolumn{10}{|l|}{ Ways of Savoring Checklist (WOSC) Subscales: } \\
\hline Broadband Dampening & 3.23 & 1.25 & 2.88 & 1.00 & 2.63 & $286^{+}$ & .009 & 0.33 & .16 \\
\hline Broadband Amplifying & 4.29 & 1.21 & 4.71 & 1.18 & 2.99 & 286 & .003 & 0.35 & .17 \\
\hline Awareness of Temporal Fleetingness \& Memory-Building & 4.27 & 1.23 & 4.66 & 1.23 & 2.65 & 286 & .009 & 0.31 & .15 \\
\hline Sensory-Perceptual Sharpening \& Absorption & 4.01 & 1.26 & 3.95 & 1.08 & 0.46 & 286 & .647 & 0.05 & .03 \\
\hline Behavioral Expression & 3.95 & 1.58 & 4.28 & 1.63 & 1.77 & 286 & .078 & 0.21 & .10 \\
\hline Sharing with Others & 4.82 & 1.44 & 5.38 & 1.30 & 3.45 & 286 & .001 & 0.41 & .20 \\
\hline
\end{tabular}

Note: ${ }^{+}$Gender comparisons for the SBI Rejection of Cognitive Elaboration and WOSC Broadband Dampening subscales were analyzed using Welch's $t$-test, rather than Student's $t$-test, because of significant gender differences in the variances of these two dependent measures. $M=$ mean. $S D=$ standard deviation. 
Table 4. Conformity to collectivist social norms and rejection of traditional Asian values as mediators of the effect of gender on savoring responses $(N=288)$

\begin{tabular}{|c|c|c|c|c|c|c|c|c|c|}
\hline \multicolumn{4}{|l|}{ Mediator: Conformity to Collectivist Social Norms } & \multicolumn{6}{|c|}{ Indirect (Mediated) Effect } \\
\hline \multirow[b]{2}{*}{ Savoring Responses } & \multicolumn{3}{|c|}{ Coefficients } & \multirow[b]{2}{*}{$\begin{array}{c}\text { Point } \\
\text { Estimate }\end{array}$} & \multirow[b]{2}{*}{$S E$} & \multirow[b]{2}{*}{$\beta$} & \multirow[b]{2}{*}{$Z$} & \multicolumn{2}{|c|}{$\begin{array}{c}\text { Bootstrapped } \\
\text { BC } 95 \% \text { CI } \\
\end{array}$} \\
\hline & $a$ & $b$ & $c$ & & & & & Lower & Upper \\
\hline Broadband Dampening & $-.50^{* * * *}$ & $.18^{*}$ & -.26 & -.09 & .04 & -.04 & $2.23^{*}$ & -.19 & -.03 \\
\hline Broadband Amplifying & $-.50^{* * *}$ & $.15^{*}$ & $.50^{* * *}$ & -.07 & .04 & -.03 & 1.82 & -.17 & -.01 \\
\hline Awareness of Temporal Fleetingness \& Memory-Building & $-.50^{* * *}$ & .12 & $.45^{* *}$ & -.06 & .04 & -.02 & 1.50 & -.16 & .01 \\
\hline Sensory-Perceptual Sharpening \& Absorption & $-.50^{* * *}$ & .10 & -.01 & -.05 & .04 & -.02 & 1.28 & -.14 & .03 \\
\hline Behavioral Expression & $-.50^{* * *}$ & $-.24^{*}$ & .22 & .12 & .06 & .04 & $2.12^{*}$ & .02 & .25 \\
\hline Sharing with Others & $-.50^{* * *}$ & -.07 & $.52^{* *}$ & .03 & .04 & .01 & 0.79 & -.06 & .14 \\
\hline \multicolumn{4}{|l|}{ Mediator: Rejection of Traditional Asian Values } & \multicolumn{6}{|c|}{ Indirect (Mediated) Effect } \\
\hline \multirow[b]{2}{*}{ Savoring Responses } & \multicolumn{3}{|c|}{ Coefficients } & \multirow[b]{2}{*}{$\begin{array}{c}\text { Point } \\
\text { Estimate }\end{array}$} & \multirow[b]{2}{*}{$S E$} & \multirow[b]{2}{*}{$\beta$} & \multirow[b]{2}{*}{$Z$} & \multicolumn{2}{|c|}{$\begin{array}{c}\text { Bootstrapped } \\
\text { BC } 95 \% \text { CI }\end{array}$} \\
\hline & $a$ & $b$ & $c$ & & & & & Lower & Upper \\
\hline Broadband Dampening & $.44^{* * *}$ & $-.39^{* * *}$ & -.18 & -.17 & .05 & -.08 & $3.38^{* * * *}$ & -.31 & -.08 \\
\hline Broadband Amplifying & $.44^{* * *}$ & $-.14^{+}$ & $.48^{* *}$ & -.06 & .04 & -.03 & 1.55 & -.16 & .01 \\
\hline Awareness of Temporal Fleetingness \& Memory-Building & $.44^{* * *}$ & -.09 & $.42^{* *}$ & -.04 & .04 & -.02 & 1.01 & -.14 & .04 \\
\hline Sensory-Perceptual Sharpening \& Absorption & $.44^{* * *}$ & $-.24^{* *}$ & .04 & -.11 & .04 & -.05 & $2.47^{*}$ & -.22 & -.03 \\
\hline Behavioral Expression & $.44^{* * *}$ & -.03 & $.35^{+}$ & -.01 & .05 & -.01 & 0.22 & -.13 & .10 \\
\hline Sharing with Others & $.44^{* * *}$ & $.18^{+}$ & $.48^{* *}$ & .08 & .05 & .03 & 1.71 & -.01 & .19 \\
\hline
\end{tabular}

Note: ${ }^{t} p<.10 .{ }^{*} p<.05 .{ }^{* *} p<.01 .{ }^{* * *} p<.001 . a=$ unstandardized path coefficient using gender $(0=$ male, $1=$ female $)$ to predict the mediator. $b=$ unstandardized path coefficient using the mediator to predict savoring responses. $c=$ unstandardized path coefficient using gender to predict savoring responses when the mediator is included in the path model. $S E=$ standard error. $\beta=$ standardized indirect effect. $\mathrm{BC}=$ bias corrected, using 5,000 bootstrap resamples. $\mathrm{CI}=$ confidence interval. In some cases, the product of the tabled values of coefficients $a$ and $b$ diverges slightly from the tabled value of the point estimate of the indirect effect due to rounding error. 
Figure 1. Mediation model for associations between gender and broadband dampening as mediated by rejection of traditional Asian values $(N=288)$

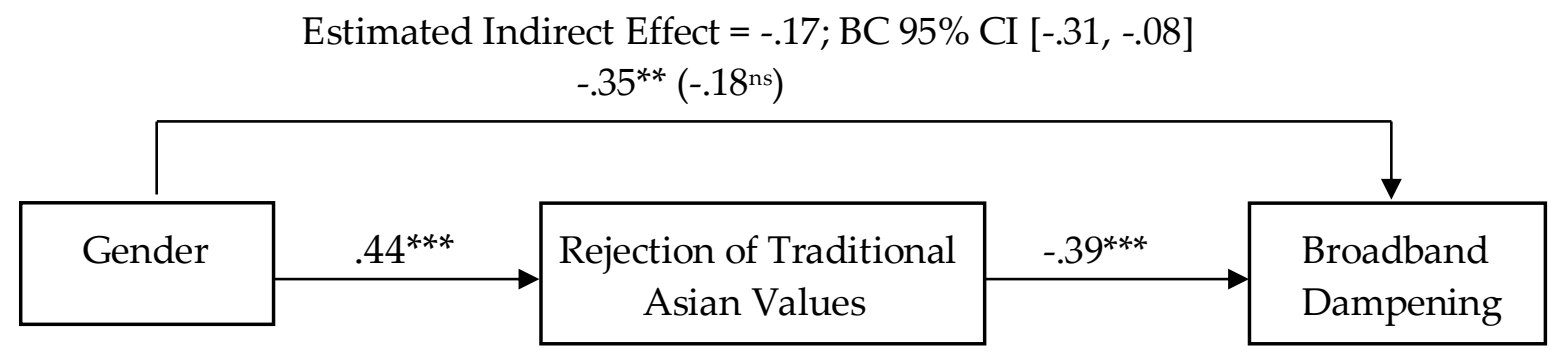



Note: Values displayed above pathways are standardized regression coefficients. The first value above the pathway linking gender to broadband dampening represents the standardized regression coefficient when ignoring the mediator, whereas the value in parentheses represents the standardized regression coefficient when controlling for the mediator. BC: Bias Corrected; 5,000 bootstrap samples. CI = confidence interval. Gender was coded as male $=0$ and female $=1$. Gender explained $6 \%$ of the variance in Rejection of Traditional Asian Values, which in turn explained 11\% of the variance in Broadband Dampening.

Rejection of traditional Asian values mediated $49 \%$ of the total effect of gender on dampening. On the other hand, although the results for Hypothesis 3 above highlighted significant gender differences in the use of amplifying strategies (see Table 3), tests of indirect effects revealed that the rejection of traditional Asian values was only marginally associated with greater amplifying $(\beta=-.03, p<.10)$ and did not significantly mediate the influence of gender on amplifying (see Table 4 ).

\section{Discussion}

Relevant to the fields of positive psychology and cross-cultural psychology, the current study enhances our understanding of the relationships among gender, cultural values, and savoring in Korea. Savoring beliefs and responses are core constructs in the field of psychology (Bryant \& Veroff, 2007), but are novel conceptual frameworks within Korean culture. Given the absence of research on Koreans' experiences of savoring, our results contribute to an understanding of how culture influences the ways in which individuals approach positive events and regulate their positive emotions in response to these events.

Paralleling Eagly and Wood's (1999) work on gender differences in emotional experience, Bryant and Veroff (2007) reported gender differences among Western samples in savoring responses to positive events, whereby males are more likely to use kill-joy thinking, a dampening strategy, and females are more likely to endorse cognitive and behavioral amplifying strategies. Replicating findings from these Western samples, the present Korean sample showed the same pattern of gender differences in broadband dampening and amplifying responses. Korean women, relative to men, reported greater savoring ability, and used less dampening and more amplifying of emotional responses in reaction to positive events. Moreover, our findings suggest that females' greater rejection of traditional Asian values at least partially underlies the gender difference in the dampening of positive emotion that exists within Korean culture.

The present study illuminates several important conclusions with respect to the influence of culture on savoring. In particular, our results indicate that cultural values shape (a) personal beliefs about savoring capacity and (b) the specific savoring strategies individuals use in response to positive events. 
What implications do these research results have for understanding cross-cultural differences in response to positive emotions? First, our results support the notion that traditional Asian values place less emphasis on the pursuit and attainment of happiness, as has been highlighted in prior cross-cultural research on positive emotions (e.g., Eid \& Diener, 2001; Joshanloo \& Weijers, 2014; Kitayama, Mesquita, \& Karasawa, 2006). Our findings are also consistent with the view that aversion to happiness is shaped by cultural norms (Joshanloo \& Weijers, 2014). In particular, dampening responses in reaction to pleasant events tend to reduce and curtail happiness (Jose et al., 2012). Thus, rejecting traditional cultural values evidently leads Korean women to respond to positive events in ways that are less antagonistic toward positive feelings.

Concerning the rejection of traditional Asian values, theorists have argued that the influence of Confucian norms is destined to decline as globalization increases (see Rozman, 2002). Along these lines, we can speculate that the gender differences in dampening that we have observed among Koreans may well disappear over time, if males eventually become less traditional in their values, as females appear to be. In fact, research has documented a similar pattern of sociohistorical change in the meaning of subjective wellbeing in the United States between the 1950s and 1970s, as men shifted away from a traditional emphasis on career toward an emphasis on family as a basis for gauging quality of life, and became more similar to women in the structure of their self-evaluations (Bryant \& Veroff, 1982).

Our findings indicate that Korean males dampen positive emotions in response to pleasant events more strongly than do Korean females, even after accounting for differences in the degree to which they endorse traditional Asian values. Specifically, level of belief in traditional Asian values explains roughly half of the relationship between gender and dampening in our Korean sample. Thus, about half of the total effect of gender on dampening is mediated by other factors besides traditional Asian values.

In interpreting the observed gender differences in savoring beliefs and savoring responses, it is important to distinguish between inner cognitive and emotional experience and the outward expression of inner experience (Brody \& Hall, 1993). The literature on emotion suggests that gender differences (i.e., females stronger than males) are generally more pronounced for emotional expression than for inner experience. Furthermore, within the expressivity domain, males tend to express emotions primarily in terms of physiological reactions and overt behavior, whereas females more often express emotions through verbal communication and nonverbal expressions that reflect a more conscious awareness of emotion (Brody \& Hall, 1993). Thus, women may be more aware of emotional reactions and more willing and able to express them, relative to men (Bryant, Yarnold, \& Grimm, 1996). Furthermore, the gender differences we have found in savoring beliefs and savoring responses may simply reflect a gender difference in response styles rather than a difference in inner experience. For example, women may be more likely than men to make public affirmations of positive emotions of greater variety and intensity, due to gender-based emotional display rules (LaFrance \& Banaji, 1992).

Although Korean respondents understood and could relate to the Western paradigm of savoring beliefs and responses, they also showed a distinct pattern of responses with respect to savoring, compared to the patterns observed in research with American participants (Bryant \& Veroff, 2007). Korean women rejected traditional cultural values more strongly than did Korean men. Although this rejection of traditional values mediated the relationship between being female and engaging in less Eastern-style dampening in response to positive events, it did not mediate the relationship between being female and engaging in greater Western-style amplifying in response to positive events. This pattern of results indicates that despite females' greater 
rejection of traditional cultural values, culture continues to shape their savoring responses in ways that remain normatively appropriate in Korean society-specifically, the rejection of traditional Asian values was not associated with great amplifying of positive emotions.

The present study also contributes to the research literature on cross-cultural positive psychology through the development and preliminary validation of Korean versions of the Savoring Beliefs Inventory and the Ways of Savoring Checklist. In the current study, it was vital to translate English-language materials into the Korean language by the process of backtranslation, in order to resolve conceptual discrepancies or ambiguities of cross-cultural translation and thereby ensure the construct validity of the dependent measures (Brislin, 1970). The availability of these measurement tools should facilitate future research, not only investigating the dynamics of savoring in the context of Korean culture, but also comparing savoring beliefs and processes more directly across Korean and Western cultures.

Several limitations to the present study suggest potentially fruitful directions for future research. First, we used only self-reported measures of cultural values, savoring beliefs, and responses. For example, the Ways of Savoring Checklist (Bryant \& Veroff, 2007) was used to assess savoring responses, some of which reflect behavioral reactions to actual life events. There might, however, be discrepancies between self-reported behaviors and observable behavioral responses to such events. Future researchers should develop and use behavioral measures of savoring responses, in order to overcome this limitation.

The present study also relied on measures of savoring beliefs and responses that were originally developed using Western samples (Bryant, 2003; Bryant \& Veroff, 2007) to assess these constructs in a Korean sample. Although this assessment approach yielded a set of reliable composite measures that partially resembled those found in Western samples, it did not allow us to measure savoring beliefs and responses that are unique to Korean culture. Future research has much to gain potentially from developing measures of savoring beliefs and responses that are specific to Korean culture, rather than adopting measures of these constructs that are grounded in the perspectives of Western culture. In particular, whereas the Ways of Savoring Checklist includes items reflecting only a single dimension of cognitive dampening (i.e., "KillJoy Thinking"), there may well be multiple dimensions of positive emotional dampening, e.g., distraction, fault-finding, externalization, suppression (Quoidbach, Berry, Hansenne, \& Mikolajczak, 2010), that Koreans use to dampen positive emotions in response to positive events. Assessing savoring responses that are specific to Korean culture might facilitate a clearer and more in-depth understanding of the dynamics of savoring among Koreans.

Another important limitation to our research conclusions involves our use of exploratory factor analysis to develop subscales assessing Asian values, savoring beliefs, and ways of savoring without a separate replication. Clearly, future factor analytic studies should examine these subscales using larger, independent samples.

In addition, the current study used a cross-sectional research design that is inherently correlational in nature. Future work might adopt a longitudinal design to study relationships among key study variables over time and examine the relationship between savoring and subjective wellbeing prospectively. Along these lines, daily diary methods (Jose et al., 2012) and experience-sampling procedures (Bryant et al., 2011) may prove valuable in clarifying the influence of savoring strategies on both momentary positive affect and longer-term life quality.

Although we measured the degree to which participants endorsed traditional Asian values, it should be noted that we did not assess the degree to which participants endorsed Western values. Future research might include separate measures of Western cultural values along with measures of Asian cultural values, in order to determine whether or not the rejection of 
traditional Asian values found among Korean women is accompanied by greater endorsement of Western values.

\section{Authors}

Soyeon Kim

Loyola University Chicago

skim29@luc.edu

Fred B. Bryant

Loyola University Chicago

\section{Publishing Timeline}

Received 25 February 2017

Accepted 12 September 2017

Published 25 November 2017

\section{References}

Beck, A. T. (1975). Cognitive therapy and the emotional disorders. New York, NY: International Universities Press.

Brislin, R. W. (1970). Back-translation for cross-cultural research. Journal of Cross-Cultural Psychology, 1, 185-216. https://doi.org/10.1177/135910457000100301

Brody, L. R., \& Hall, J. A. (1993). Gender and emotion. In M. Lewis \& J. Haviland (Eds.), Handbook of emotions (pp. 447-461). New York, NY: Guilford.

Bryant, F. B. (1989). A four-factor model of perceived control: Avoiding, coping, obtaining, and savoring. Journal of Personality, 57, 773-797. https://doi.org/10.1111/j.1467-6494.1989.tb00494.x

Bryant, F. B. (2003). Savoring Beliefs Inventory (SBI): A scale for measuring beliefs about savoring. Journal of Mental Health, 12, 175-196. https://doi.org/10.1080/0963823031000103489

Bryant, F. B., Chadwick, E. D., \& Kluwe, K. (2011). Understanding the processes that regulate positive emotional experience: Unsolved problems and future directions for theory and research on savoring. International Journal of Wellbeing, 1, 107-126. https://doi.org/10.5502/ijw.v1i1.18

Bryant, F. B., \& Veroff, J. (1982). The structure of psychological well-being: A sociohistorical analysis. Journal of Personality and Social Psychology, 43, 653-673. https://doi.org/10.1037/0022-3514.43.4.653

Bryant, F. B., \& Veroff, J. (2007). Savoring: A new model of positive experience. Mahwah, NJ: Lawrence Erlbaum Associates.

Bryant, F. B., Yarnold, P. R., \& Grimm, L. G. (1996). Toward a measurement model of the Affect Intensity Measure: A three-factor structure. Journal of Research in Personality, 30, 223-247. https://doi.org/10.1006/jirpe.1996.0015

Deuchler, M. (1993). The Confucian transformation of Korea: A study of society and ideology. Cambridge, MA: Harvard University Press.

Dinno, A. (2009). Exploring the sensitivity of Horn's parallel analysis to the distributional form of random data. Multivariate Behavioral Research, 44, 362-388. https://doi.org/10.1080/00273170902938969

Eagly, A. H., \& Wood, W. (1982). Inferred sex differences in status as a determinant of gender stereotypes about social influence. Journal of Personality and Social Psychology, 43, 915-928. https://doi.org/10.1037/0022-3514.43.5.915

Eagly, A. H., \& Wood, W. (1999). The origins of sex differences in human behavior: Evolved dispositions versus social roles. American Psychologist, 54, 408-423. https://doi.org/10.1037/0003-066X.54.6.408

Eid, M., \& Diener, E. (2001). Norms for experiencing emotions in different cultures: Inter- and intranational differences. Journal of Personality and Social Psychology, 81, 869-885.

https://doi.org/10.1037/0022-3514.81.5.869 
Grossman, M., \& Wood, W. (1993). Sex differences in intensity of emotional experience: A social role interpretation. Journal of Personality and Social Psychology, 65, 1010-1022. https://doi.org/10.1037/0022$\underline{3514.65 .5 .1010}$

Horn, J. L. (1965). A rationale and test for the number of factors in factor analysis. Psychometrika, 30, 179185. https://doi.org/10.1007/BF02289447

Jose, P. E., Lim, B. T., \& Bryant, F. B. (2012). Does savoring increase happiness? A daily diary study. Journal of Positive Psychology, 7, 176-187. https://doi.org/10.1080/17439760.2012.671345

Joshanloo, M. (2014). Eastern conceptualizations of happiness: Fundamental differences with Western views. Journal of Happiness Studies, 15, 475-493. https://doi.org/10.1007/s10902-013-9431-1

Joshanloo, M., Lepshokova, Z. K., Panyusheva, T., Natalia, A., Poon, W.-C., Yeung, V. W., \& Jian, D.-Y. (2014). Cross-cultural validation of Fear of Happiness Scale across 14 national groups. Journal of Cross-Cultural Psychology, 45, 246-264. https://doi.org/10.1177/0022022113505357

Joshanloo, M., \& Weijers, D. (2014). Aversion to happiness across cultures: A review of where and why people are averse to happiness. Journal of Happiness Studies, 15, 717-735. https://doi.org/10.1007/s10902-013-9489-9

Kalton, M. C. (1994). Review of "The Confucian transformation of Korea: A study of society and ideology" by M. Deuchler. The Journal of Asian Studies, 53, 239-240. https://doi.org/10.2307/2059601

Kim, B. S., Atkinson, D. R., \& Yang, P. H. (1999). The Asian Value Scale: Development, factor analysis, validation, and reliability. Journal of Counseling Psychology, 46, 342-352. https://doi.org/10.1037/0022$\underline{0167.46 .3 .342}$

Kim, B. S. K., Yang, P. H., Atkinson, D. R., Wolfe, M. M., \& Hong, S. (2001). Cultural value similarities and differences among Asian American ethnic groups. Cultural Diversity \& Ethnic Minority Psychology, 7, 343-361. https://doi.org/10.1037/1099-9809.7.4.343

Kim, S. C. (1997). Korean American families. In E. Lee (Ed.), Working with Asian Americans (pp. 125-135). New York, NY: The Guilford Press.

Kitayama, S., Mesquita, B., \& Karasawa, M. (2006). Cultural affordances and emotional experience: Socially engaging and disengaging emotions in Japan and the United States. Journal of Personality and Social Psychology, 91, 890-903. https://doi.org/10.1037/0022-3514.91.5.890

Kwon, S., Lim, Y, \& Ha, S. (2010). 인생을 향유하기 (Savoring: A new model of positive experience). Seoul, Korea: Hakjisa.

LaFrance, M., \& Banaji, M. (1992). Toward a reconsideration of the gender-emotion relationship. In M. S. Clark (Ed.), Emotion and social behavior (pp. 178-201). Newbury Park, CA: Sage.

Miyamoto, Y., \& Ma, X. (2011). Dampening or savoring positive emotions: A dialectical script guides emotion regulation. Emotion, 1, 1346-1357. https://doi.org/10.1037/a0025135

Matsumoto, D., Yoo, S. H., Fontaine, J., Anguas-Wong, A. M., Ariola, M., Ataca, B., \& Grossi, E. (2008). Mapping expressive differences around the world: The relationship between emotional display rules and individualism versus collectivism. Journal of Cross-Cultural Psychology, 39, 55-74. https://doi.org/10.1177/0022022107311854

Matsunaga, M. (2010). How to factor-analyze your data right: Do's, don'ts, and how-to's. International Journal of Psychological Research, 3, 97-110. https://doi.org/10.21500/20112084.854

Park, I. H., \& Cho, L.-J. (1995). Confucianism and the Korean family. Journal of Comparative Family Studies, $26,117-134$.

Preacher, K. J., \& Hayes, A. F. (2008). Asymptotic and resampling strategies for assessing and comparing indirect effects in multiple mediator models. Behavior Research Methods, 40, 879-891.

Quoidbach, J., Berry, E., Hansenne, M., \& Mikolajczak, M. (2010). Positive emotion regulation and wellbeing: Comparing the impact of eight savoring and dampening strategies. Personality and Individual Differences, 49, 368-373. https://doi.org/10.1016/j.paid.2010.03.048

Rosaldo, M. Z., \& Lamphere, L. (1974). Introduction. In M. Z. Rosaldo \& L. Lamphere (Eds.), Woman, culture and society (pp. 1-16). Stanford, CA: Stanford University Press.

Rozman, G. (2002). Can Confucianism survive in an age of universalism and globalization? Pacific Affairs, 75, 11-37. https://doi.org/10.2307/4127239 
Seligman, M. E. P., \& Csikszentmihalyi, M. (2000). Positive psychology: An introduction. American Psychologist, 55, 5-14. https://doi.org/10.1037/0003-066X.55.1.5

Smith, J. L., \& Bryant, F. B. (2012). Are we having fun yet? Savoring, Type A behavior, and vacation enjoyment. International Journal of Wellbeing, 3, 1-19. https://doi.org/10.5502/ijw.v3i1.1

Smith, J. L., \& Bryant, F. B. (2017). Savoring and well-being: Mapping the cognitive-emotional terrain of the happy mind. In M. D. Robinson \& M. Eid (Eds.), The happy mind: Cognitive contributions to wellbeing (pp.139-156). New York, NY: Springer. https://doi.org/10.1007/978-3-319-58763-9 8

Stacki, S. L., \& Monkman, K. (2003). Change through empowerment processes: Women's stories from South Asia and Latin America. Compare, 33, 173-189.

Thompson, B. (2004). Exploratory and confirmatory factor analysis: Understanding concepts and applications. Washington, DC: American Psychological Association. https://doi.org/10.1037/10694-000

Uchida, Y., Norasakkunkit, V., \& Kitayama, S. (2004). Cultural constructions of happiness: Theory and empirical evidence. Journal of Happiness Studies, 5, 223-239. https://doi.org/10.1007/s10902-004-8785-9

Uchida, Y., \& Kitayama, S. (2009). Happiness and unhappiness in east and west: Themes and variations. Emotion, 9, 441-456. https://doi.org/10.1037/a0015634

Velicer, W. F., \& Fava, J. L. (1998). Effects of variable and subject sampling on factor pattern recovery. Psychological Methods, 3, 231-251. https://doi.org/10.1037/1082-989X.3.2.231

Wood, W., \& Eagly, A. H. (2002). A cross-cultural analysis of the behavior of women and men: Implications for the origins of sex differences. Psychological Bulletin, 128, 699-727. https://doi.org/10.1037/0033-2909.128.5.699

Young, F. W., Valero-Mora, P., \& Friendly, M. (2006). Visual statistics: Seeing data with dynamic interactive graphics. Hoboken, NJ: Wiley and Sons. https://doi.org/10.1002/9781118165409 